\title{
Predictive formulas expressing relationship between dose rate and survival time in total body irradiation in mice
}

\author{
Sung Jang Chung \\ Morristown-Hamblen Healthcare System, Morristown, USA. \\ Email: $\underline{\text { sung.chung@,comcast.net }}$
}

Received 10 September 2011; revised 4 October 2011; accepted 24 October 2011.

\begin{abstract}
The Gompertz model is the long-time well-known mathematical model of exponential expression among mortality models in the literature that are used to describe mortality and survival data of a population. The death rate of the "probacent" model developed by the author based on animal experiments, clinical applications and mathematical reasoning was applied to predict age-specific death rates in the US elderly population, 2001, and to express a relationship among dose rate, duration of exposure and mortality probability in total body irradiation in humans. The results of both studies revealed a remarkable agreement between "probacent"-formula-predicted and published-reported values of death rates in the US elderly population or mortality probabilities in total body irradiation in humans ( $p$-value $>0.995$ in $\chi^{2}$ test in each study). In this study, both the Gompertz and "probacent" models are applied to the Sacher's comprehensive experimental data on survival times of mice daily exposed to various doses of total body irradiation until death occurs with an assumption that each of both models is applicable to the data. The purpose of this study is to construct general formulas expressing relationship between dose rate and survival time in total body irradiation in mice. In addition, it is attempted to test which model better fits the reported data. The results of the comparative study revealed that the "probacent" model not only fit the Sacher's reported data but also remarkably better fit the reported data than the Gompertz model. The "probacent" model might be hopefully helpful in research in human tolerance to low dose rates for long durations of exposure in total body irradiation, and further in research in a variety of biomedical phenomena.
\end{abstract}

Keywords: Lethal Radiation Dose; Total Body Irradiation; Formula of Survival Time in Mice; Dose-Survival
Curve; "Probacent" Model; Gompertz Model

\section{INTRODUCTION}

The Gompertz model (1825) is the long-time well-known mathematical model of exponential expression among mortality models in the literature that are used to describe mortality and survival data of a population [1-3].

The ordinary procedure in biomedical survival data analysis is to apply the non-parametric life table method or nowadays, especially the non-parametric KaplanMeier product-limit method [1,4-8].

The most commonly used methods of parametric estimation for distributions of survival times are the fittings of exponential, lognormal, Weibull, gamma and Gompertz function of survival time.

\subsection{The "Probacent" Model}

On the basis of experimental observations on animals, clinical applications and mathematical reasoning, the author developed a general mathematical model of "probacent"-probability equation that may be applicable as a general approximation method to possibly calculate the probability of safe survival in humans and other living organisms exposed to any harmful or adverse circumstances in overcoming the risk, and further to predict degrees of risk and/or mortality probability in terms of percent probability. In this way, the "probacent" model might make useful predictions of probable outcomes in a variety of biomedical phenomena in protecting exposed subjects [9-12].

The model of "probacent"-probability equation expressed by Eq.1 was constructed from experimental studies on animals to express survival probability in mice exposed to $g$-force in terms of magnitude of acceleration and exposure time $[9,13]$; and to express a relationship among intensity of stimulus or environmental agent (such as drug [9,10,14], heat [15], pH [16], and electroshock $[15,17])$, duration of exposure and biological response in animals. 
The model has been applied to data in the literature to express a relationship among dose rate, duration of exposure and mortality probability in total body irradiation in humans $[12,18]$; to express carboxyhemoglobin levels of blood as a function of carbon monoxide concentration in air and duration of exposure [19,20]; to express a relationship among plasma acetaminophen concentration, time after ingestion and occurrence of hepatotoxicity in man $[21,22]$; to predict survival probability in patients with malignant melanoma [23,24]; to predict survival probability in patients with heart transplantation [25]; to express a relationship among age, height and weight, and percentile in Saudi and US children of 6 - 16 years of age $[26,27]$; to predict the percentile of heart weight by body weight from birth to 19 years of age [28,29]; and to predict the percentile of serum cholesterol levels by age in adults $[30,31]$.

The model was applied to the United States life tables, 1992 and 2001 reported by the National Center for Health Statistics (NCHS) to construct formulas expressing age-specific survival probability, death rate and life expectancy in US adults, men and women [11,32-34].

The formula of survival probability is expressed by the following "probacent"-probability equation, Eq.1.

$$
\begin{gathered}
P^{\gamma}=A-B \log T \\
S=\frac{10}{\sqrt{2 \pi}} \int_{-\infty}^{P} \exp \left[-\frac{(P-50)^{2}}{200}\right] \mathrm{d} P
\end{gathered}
$$

where $T=$ time after biomedical insult, diagnosis of cancer or age; $P=$ "probacent" (abbreviation of probability percentage) $=$ relative biological amount of "reserve" for survival; "probacent" $(P)$ of 0,50 and 100 corresponds to mean - 5 S.D., mean and mean + 5 S.D., respectively; one "probacent" is equivalent to 0.1 S.D. in a normal distribution. In addition, 0, 50 and 100 "probacent" seem to correspond to 0,50 and 100 percent probability in mathematical prediction problems in terms of percentage. Therefore, the survival probability could be used to predict probabilities in general biomedical phenomena. "probacent" values are obtainable from a list of conversion of percent probability into "probacent" that was published by the author (Table 6 of Ref. [9] and Table 4 of Ref. [10]). $\gamma, A$ and $B$ are constants; $A$ is an intercept and $B$ a slope; $\gamma$ represents a curvature (a shape of a curve) and expressed by the following equation:

$$
\gamma=\log (A-B \log T) / \log P
$$

If the value of $\gamma$ becomes equal to one, Eq.1 represents a log-normal distribution. Eq.1 is considered to be fundamentally based on the Gaussian normal distribution.
Eq.2 representing death rate is derived from Eq.1 expressing survival probability [33].

$$
(\log D)^{c}=a+b \log T
$$

where $D$ represents death rate in percentage (mortality probability); $T$ is time or age; $c, a$ and $b$ are constants; $c$ represents a curvature (a shape of curve) like $\gamma$ in Eq.1a; $\mathrm{a}$ is an intercept and b a slope.

If the value of constant $c$ becomes equal to one, Eq.2 is essentially similar to the Weibull distribution [1].

Eq.2 was applied to express death rates in US adults $[11,33,34]$. It was found to better express death rates in US elderly population than the Gompertz, the exponential and the Weibull distributions [11].

Mehta and Joshi [35] successfully applied the "probacent"-probability equation, Eqs.1 and 2 to use modelderived data as an input for radiation risk evaluation of Indian population.

A clear and exact quantitative relationship between dose of radiation and mortality in humans is still not known because of lack of human data that would enable to determine $\mathrm{LD}_{50}$ for humans in total body irradiation. Analysis of human data has been primarily from radiation accidents, radiotherapy and the atomic bomb victims. Consequently, laboratory animals have been used to investigate the relationship between radiation dose and effect in total body irradiation and further to possibly derive a general predictive formula [9,36-40].

The author has applied Eq.2 to predict mortality probability in total body irradiation without medical support in humans as a function of dose rate and duration of exposure [12]; the formula of the function is constructed on the basis of animal-model predicted data published by Cerveny, MacVittie and Young [18]. There is a remarkable agreement between formula-predicted and published estimated $\mathrm{LD}_{50}$ and also between both mortality probabilities ( $p$ value $>0.995)$.

\subsection{The Gompertz Modelod}

Helligman and Pollard [2] reported that the Gompertz model applied to the Australian national mortality data required a mathematical modification for a curvature noticed in his graphical analysis of the older age population.

Sacher published a comprehensive experimental data on daily dose rates versus average survival times in total body irradiation in mice $[36,37]$. He stated in his discussion that the Gompertz model seemed to be approximately applicable to the data on dose rates versus death rates in mice. However, he did not present a general formula of the Gompertz model in his articles [36,37].

The purpose of this study is to apply each of both the "probacent" and the Gompertz models to the Sacher's 
reported data above described with an assumption that each model is applicable to the data, and to test which of the models would better fit the reported data on doses versus survival times in mice on the basis of results of statistical analysis.

\section{MATERIALS AND METHODS}

Sacher of Argonne National Laboratory, USA reported his comprehensive experimental data on mean aftersurvival times (MAS) of adult LAF1 mice irradiated daily with various doses for the duration of life, beginning at 100 days of age until deaths occurred [36,37]. The total number of mice was 4692; 2348 male and 2344 female mice. The range of dose rate is from 6 to 2500 $\mathrm{r}$ /day. The MAS is from 5 to 548 days; 5 - 6 days at high dose rates ( $\geq 1100 \mathrm{r} /$ day) and 501 - 548 days at the lowest dose rate, $6 \mathrm{r} /$ day.

The author used the Sacher's reported data [37] to construct general formulas to express relationships between dose rates and mean after-survival times in total body irradiation of $\gamma$-ray in male and female mice.

The data on the relationship between dose rate and mean after-survival time in both male and female mice are shown in Table $\mathbf{1}$ and plotted on a log-log graph paper as illustrated in Figure $\mathbf{1}$ to improve the overall fit in mathematical analysis.

A closer look at the lines connecting data points, closed and open circles in Figure 1, respectively suggests that there appear to exist roughly four different periods of the first two weeks after beginning of radiation (range of $2500-330 \mathrm{r} /$ day) in an acute period, two weeks to one month (range: 330 - $125 \mathrm{r} /$ day) in an early subacute period, one to five months (range: 125 - 43 $\mathrm{r}$ /day) in a late subacute period, and five to 18 months (range: 43 - $6 \mathrm{r} /$ day) in a chronic period. Each period would have different values of constants in the "probacent" and Gompertz models.

Both models are applied to the Sacher's data with an assumption that dose rates reflect death rates as shown in the relationship among dose rate, duration of exposure and mortality probability in total body irradiation in humans [12], and that both models would be applicable to the data.

\subsection{Formulas of Mean After-Survival Time (MAS)}

The mathematical method to construct formulas of the "probacent" model is described in Appendix and the author's previous articles $[33,41]$. The best-fitting c value is determined by a statistical method of the least sum of squares of curved regression described in the author's previous publication [42]. Appendix also describes how to construct formulas of the Gompertz model.

\subsubsection{Formulas of "Probacent" Model}

Eqs.3-5 with different values of constants, $a, b$ and $c$ are constructed to express relationships between dose rate and mean after-survival time in acute, early subacute, late subacute and chronic periods in male and female mice, respectively.

$$
\begin{gathered}
(\log R)^{c}=a+b \log T \\
\log T=(1 / b) \times\left[(\log R)^{c}-a\right] \\
T=10^{\log T}
\end{gathered}
$$

where $R=$ daily dose rate in $\mathrm{r} /$ day; $T=$ mean after-survival time (MAS) in days; $\mathrm{a}, \mathrm{b}$ and $\mathrm{c}$ are constants.

The "probacent" equations for male mice.

Acute period (2500 - 330 r/day):

$$
\begin{gathered}
a=2.86009 \times 3.21748^{0.1}-1.86009 \times 2.51851^{0.1} \\
b=2.53975 \times\left(2.51851^{0.1}-3.21748^{0.1}\right) \\
c=0.1
\end{gathered}
$$

Early subacute period (330 - 125 r/day):

$$
\begin{gathered}
a=3.78787 \times 2.51851^{0.01}-2.78787 \times 2.09691^{0.01} \\
b=2.47562 \times\left(2.09691^{0.01}-2.51851^{0.01}\right) \\
c=0.01
\end{gathered}
$$

Late subacute period (125 - 43 r/day):

$$
\begin{gathered}
a=3.37092 \times 2.09691^{0.01}-2.37092 \times 1.63347^{0.01} \\
b=1.54955 \times\left(1.63347^{0.01}-2.09691^{0.01}\right) \\
c=0.01
\end{gathered}
$$

Chronic period (43 - 6 r/day):

$$
\begin{gathered}
a=5.14555 \times 1.63347^{2.83}-4.14555 \times 0.77815^{2.83} \\
b=1.90563 \times\left(0.77815^{2.83}-1.63347^{2.83}\right) \\
c=2.83
\end{gathered}
$$

The "probacent" equations for female mice.

Acute period (2500 - 330 r/day):

$$
\begin{gathered}
a=2.67383 \times 3.21748^{0.1}-1.67383 \times 2.51851^{0.1} \\
b=2.3771 \times\left(2.51851^{0.1}-3.21748^{0.1}\right) \\
c=0.1
\end{gathered}
$$

Early subacute period (330 - 125 r/day):

$$
\begin{gathered}
a=4.04791 \times 2.51851^{0.01}-3.04791 \times 2.09691^{0.01} \\
b=2.70966 \times\left(2.09691^{0.01}-2.51851^{0.01}\right) \\
c=0.01
\end{gathered}
$$


Table 1. Mean after-survival times (MAS) of male and female mice irradiated daily for duration of life, beginning at 100 days of age [37].

\begin{tabular}{|c|c|c|c|c|c|c|}
\hline \multirow{3}{*}{$\begin{array}{l}\text { Daily Dose } \\
\text { (r/day) }\end{array}$} & \multicolumn{3}{|c|}{ Male } & \multicolumn{3}{|c|}{ Female } \\
\hline & \multicolumn{2}{|c|}{ Mean After-Survival Time (days) } & \multirow{2}{*}{$\mathrm{N}^{*}$} & \multicolumn{2}{|c|}{ Mean After-Survival Time (days) } & \multirow{2}{*}{$\mathrm{N}^{*}$} \\
\hline & Formula-Derived MAS & Sacher's-Reported MAS & & Formula-Derived MAS & Sacher's-Reported MAS & \\
\hline 2500 & 4.40 & 5.26 & 9 & 4.06 & 5.06 & 9 \\
\hline 1650 & 5.40 & 5.40 & 24 & 5.06 & 5.06 & 24 \\
\hline 1100 & 6.66 & 5.85 & 24 & 6.33 & 5.48 & 24 \\
\hline 750 & 8.21 & 7.23 & 24 & 7.92 & 6.83 & 24 \\
\hline 610 & 9.23 & 9.33 & 30 & 8.98 & 9.23 & 30 \\
\hline 500 & 10.37 & 11.00 & 30 & 10.16 & 11.30 & 30 \\
\hline 410 & 11.69 & 12.23 & 30 & 11.54 & 12.50 & 30 \\
\hline 330 & 13.37 & 13.37 & 30 & 13.33 & 13.33 & 30 \\
\hline 270 & 15.99 & 14.33 & 30 & 15.70 & 14.73 & 30 \\
\hline 220 & 19.32 & 16.47 & 75 & 18.66 & 15.81 & 75 \\
\hline 170 & 24.78 & 22.08 & 105 & 23.42 & 20.15 & 105 \\
\hline 145 & 29.06 & 29.70 & 105 & 27.10 & 24.26 & 105 \\
\hline 125 & 33.89 & 33.89 & 105 & 31.18 & 31.18 & 105 \\
\hline 97 & 46.75 & 41.09 & 105 & 45.39 & 41.71 & 105 \\
\hline 85 & 55.66 & 51.92 & 105 & 55.28 & 50.54 & 105 \\
\hline 74 & 67.21 & 64.45 & 105 & 68.07 & 65.76 & 105 \\
\hline 64 & 82.43 & 80.70 & 105 & 84.78 & 86.22 & 105 \\
\hline 56 & 100.08 & 102.11 & 120 & 103.89 & 104.18 & 120 \\
\hline 49 & 122.29 & 126.99 & 135 & 127.47 & 134.17 & 135 \\
\hline 43 & 149.77 & 149.77 & 150 & 155.93 & 155.93 & 150 \\
\hline 32 & 199.09 & 206.26 & 150 & 214.33 & 223.79 & 150 \\
\hline 24 & 252.48 & 251.61 & 183 & 277.37 & 274.05 & 183 \\
\hline 12 & 387.67 & 385.64 & 183 & 431.12 & 429.85 & 183 \\
\hline 6 & 501.40 & 501.40 & 120 & 548.22 & 548.22 & 120 \\
\hline 0 & & 494.77 & 266 & & 650.36 & 262 \\
\hline$P^{* *}$ & $>0.995$ & & & $>0.995$ & & \\
\hline
\end{tabular}

$\mathrm{N}^{*}$ : number of animals; $P^{* *}: p$ value in $\chi^{2}$ goodness-of-fit test.

Late subacute period (125 - 43 r/day):

$$
\begin{gathered}
a=3.13701 \times 2.09691^{0.01}-2.13701 \times 1.63347^{0.01} \\
b=1.43051 \times\left(1.63347^{0.01}-2.09691^{0.01}\right) \\
c=0.01
\end{gathered}
$$

Chronic period (43 - $6 \mathrm{r} /$ day):

$$
\begin{gathered}
a=5.0162 \times 1.63347^{3.18}-4.0162 \times 0.77815^{3.18} \\
b=1.83143 \times\left(0.77815^{3.18}-1.63347^{3.18}\right) \\
c=3.18
\end{gathered}
$$

\subsubsection{Formulas of Gompertz Model}

Eqs.6 and 7 are essentially similar to the Gompertz 
model.

$$
\begin{gathered}
R=10^{a+b T} \\
\log R=a+b T \\
T=(-1 / b) \times(a-\log R)
\end{gathered}
$$

where $R$ represents daily dose of total body irradiation in $\mathrm{r} /$ day; $T$ is mean after-survival time (MAS) in days; $a$ and $b$ are constants; $a$ is an intercept, $b$ a slope.

Eqs.9-12 and Eqs.13-16 are constructed to express relationships between dose rate and mean after-survival time in acute, early subacute, late subacute and chronic periods in male and female mice, respectively.

The Gompertz equations for male mice.

Acute period (2500 - 330 r/day):

$$
T=11.40251 \times(3.69106-\log R)
$$

Early subacute period (330 - $125 \mathrm{r} /$ day):

$$
T=48.67127 \times(2.79321-\log R)
$$

Late subacute period (125 - $43 \mathrm{r} /$ day):

$$
T=250 \times(2.23247-\log R)
$$

Chronic period (43 - $6 \mathrm{r} /$ day):

$$
T=411.1842 \times(1.99771-\log R)
$$

The Gompertz equations for female mice.

Acute period (2500 - $330 \mathrm{r} /$ day):

$$
T=11.83152 \times(3.64515-\log R)
$$

Early subacute period (330 - $125 \mathrm{r} /$ day):

$$
T=42.337 \times(2.83335-\log R)
$$

Late subacute period (125 - $43 \mathrm{r} /$ day):

$$
T=269.179 \times(2.21274-\log R)
$$

Chronic period (43 - $6 \mathrm{r} /$ day):

$$
T=458.716 \times(1.9734-\log R)
$$

\subsection{Description of the Computer Program}

The computer programs were written in UBASIC for IBM PC microcomputer and compatibles for Eqs.1-16. In the author's previous studies, the computer program for Eq.1 used a formula of approximation instead of the integral of Eq.1b because the computer cannot perform integral [10,41]. Mathematical transformation of the formula of integral, Eq.1b to the formula of approximation in computer programming is described in the author's book [41]. In this study, computer programs are used to calculate each equation of the "probacent" and Gompertz models.

\subsection{Statistical Analysis}

A $\chi^{2}$ goodness-of-fit test (logrank test) [43] is used to test the fit of mathematical models to the data on mean after-survival times in mice in the Sacher's article [37]. The differences are considered statistically significant when $p<0.05$. The least square curved regression method described in the author's previous publication [42] is used to determine the best-fitting $c$ value in Eqs.3-5 of the "probacent" model.

\section{RESULTS}

Table 1 shows the results of formula-derived mean after-survival times (MAS) as a function of dose rate of total body irradiation in male and female mice. Table 1 also shows comparison of the formula-derived values with the Sacher's reported data on MAS [37].

Differences between both values of formula-derived and reported MAS are statistically not significant $(p>$ $0.995)$. A close agreement is seen between both values in Table 1. The maximum difference in MAS of acute period of high dose rate, $2500-330 \mathrm{r} /$ day is \pm 1 day in both male and female mice.

Figure 1 illustrates the relationship between dose rate and mean after-survival time (MAS). It seems to the author that the distribution of the Sacher's reported MAS (closed and open circles) are very close to the formuladerived curved line for male and female mice, respectively.

Table 2 shows comparison of the least sum of squares, $\Sigma(\mathrm{E}-\mathrm{O})^{2}$ and the least maximum difference, $\mathrm{I}(\mathrm{E}-\mathrm{O}) \mathrm{I}$ in the "probacent" and Gompertz models employed in analysis of the Sacher's data on dose rate versus MAS in male and female mice. Here, $\mathrm{E}$ is a formula-derived value; $\mathrm{O}$ is a Sacher's reported value.

Figures $\mathbf{2}$ and $\mathbf{3}$ illustrate relationships between dose rates and mean after-survival times, and two lines: one solid curved line expressing the "probacent" equations and the dashed straight line expressing the Gompertz equations, in male and female mice, respectively.

The least sum of squares of differences and the least maximum difference of the "probacent" model are much smaller than those of the Gompertz model as shown in Table 2. The differences are very large in the late subacute period in both male and female mice; in the same period, the solid line expressing the "probaccent" equation is remarkably curved in contrast to the dashed straight line of the Gompertz equation.

The $\chi^{2}$-test $p$ value is greater than 0.995 for the "probacent" formulas for both male and female mice, respectively. In contrast, $p$ value is less than 0.05 for the Gompertz equations for male and female mice, respectively. These results of statistical analysis seem to indicate that the Gompertz model appears oversll not applicable 


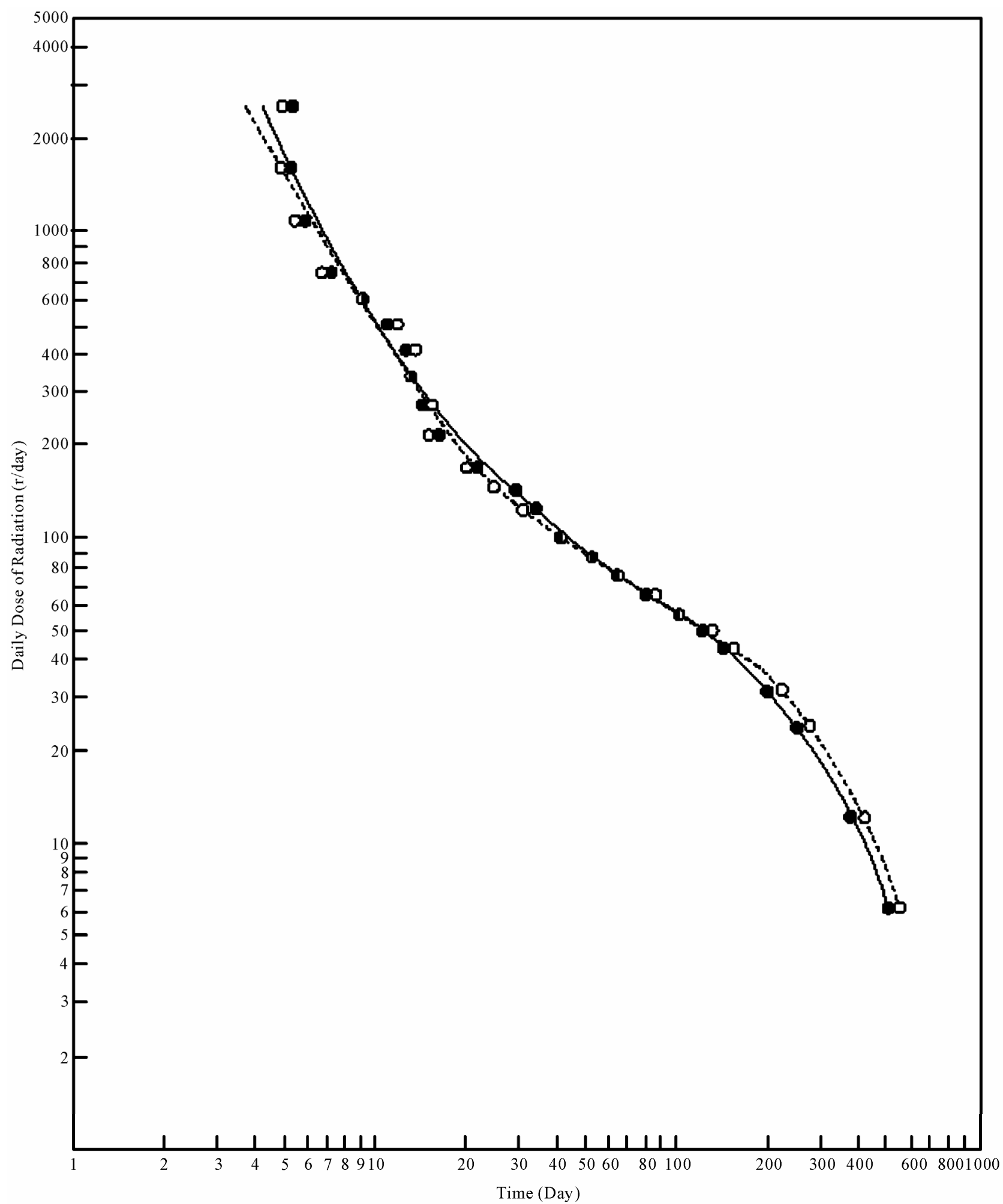

Figure 1. Relationship between dose rate of daily radiation and mean after-survival time (MAS) in LAF1 male and female mice irradiated daily during the duration of life [37]. The abscissa represents mean after-survival time in days (log scale) and the ordinate daily dose rate of radiation in $\mathrm{r}$ /day (log scale). Data points of closed and open circles indicate MAS of male and female mice, respectively. The solid and dashed curved lines represent MAS of both male and female mice, predicted by Eqs.3-5 of the "probacent" model, respectively. Data points of the Sacher's reported values appear to fall overall very close to or on the formula-derived predicted lines (see text). 


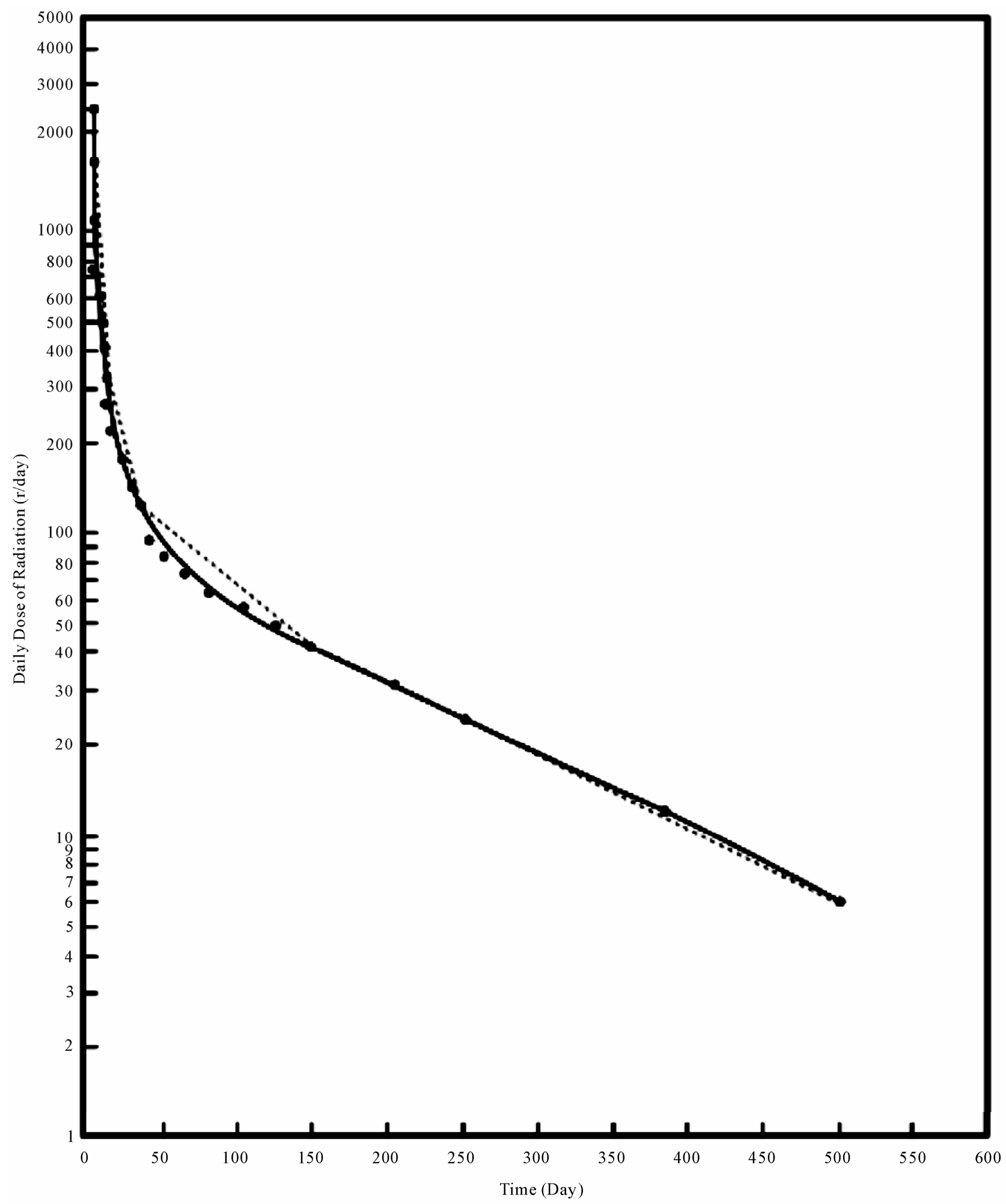

Figure 2. Relationship between dose rate and mean after-survival time (MAS) in LAF1 male mice irradiated daily during the duration of life [37]. The abscissa represents mean after-survival time in days and the ordinate daily dose rate of radiation in $\mathrm{r} / \mathrm{day}$ (log scale). Data points of closed circles indicate reported MAS of male mice. The solid, curved line and the dashed, straight lines represent MAS of male mice, predicted by the "probacent" model of Eqs.3-5 and the Gompertz model of Eqs.9-12, respectively. Data points appear to overall fall closer to or on the "probacent"-formula-predicted solid, curved line than the lines predicted by the Gompertz equations. 


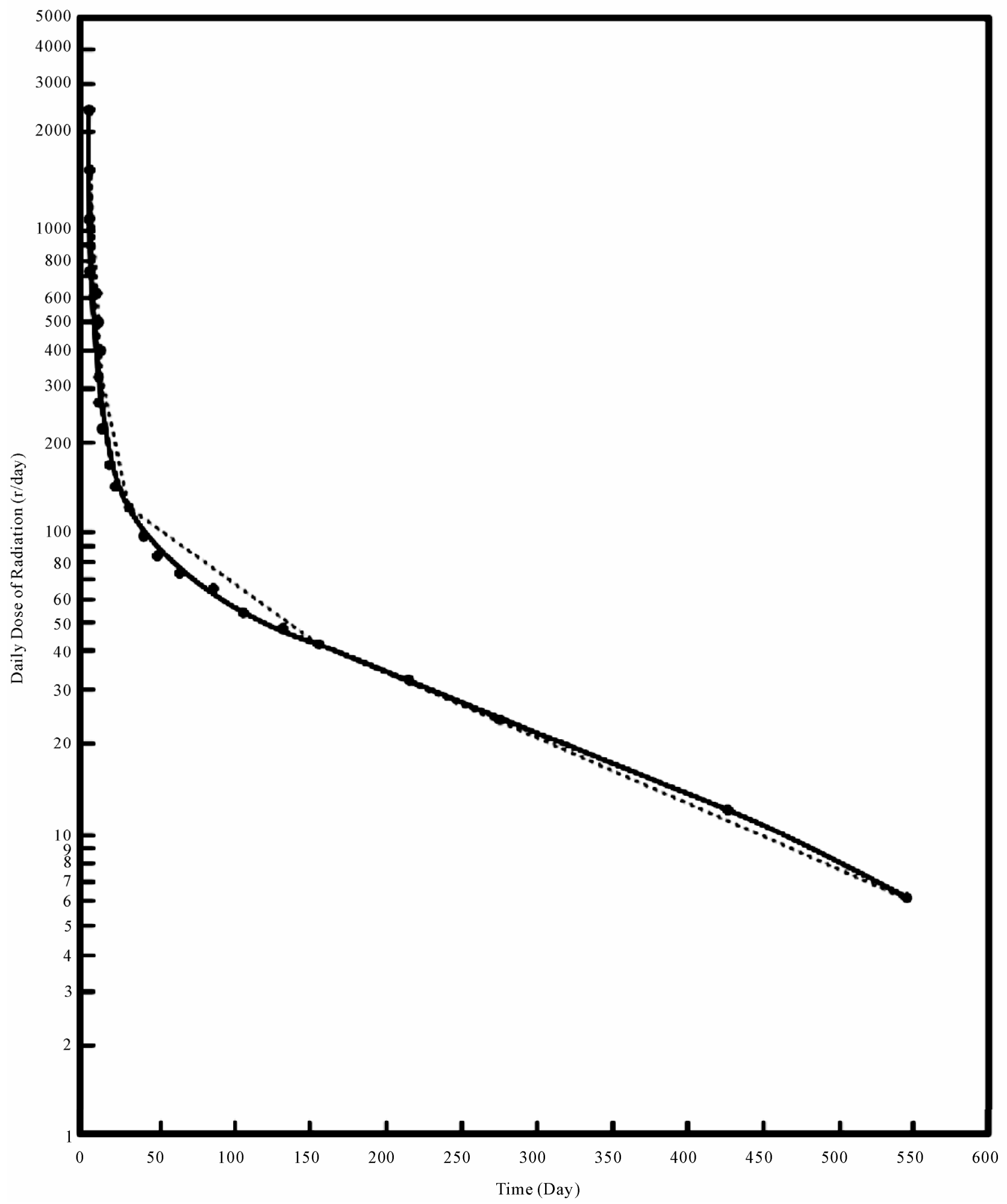

Figure 3. Relationship between dose rate and mean after-survival time (MAS) in LAF1 female mice irradiated daily during the duration of life [37]. The abscissa represents mean after-survival time (MAS) in days and the ordinate daily dose rate of radiation in r/day (log scale). Data points of closed circles indicate reported MAS of female mice. The solid, curved line and the dashed, straight lines represent MAS of female mice, predicted by the "probacent" model of Eqs.3-5, and by the Gompertz model of Eqs.13-16, respectively. Data points of the Sacher's reported values appear to overall fall closer to or on the "probacent"-formulas-predicted solid, curved line than the Gompertz-formulas-predicted dashed, straight lines. 
Table 2. Comparison of the least sum of squares, $\sum(\mathrm{E}-\mathrm{O})^{2}$ and the least maximum-difference, $\mathrm{I}(\mathrm{E}-\mathrm{O}) \mathrm{I}$, in the "probacent" and Gompertz models employed in analysis of the Sacher's data on daily radiation dose versus mean survival time in male and female mice.

\begin{tabular}{|c|c|c|c|c|}
\hline Model & & & "Probacent" & Gompertz \\
\hline \multirow{5}{*}{ Acute period } & \multicolumn{2}{|l|}{ Used equation } & Eqs.3-5 & Eqs.9,13 \\
\hline & \multirow{2}{*}{$\sum(\mathrm{E}-\mathrm{O})^{2}$} & $M^{*}$ & 3.07 & 11.50 \\
\hline & & $\mathrm{F}^{* *}$ & 5.18 & 13.51 \\
\hline & \multirow{2}{*}{$\mathrm{I}(\mathrm{E}-\mathrm{O}) \mathrm{I}$} & M & 0.98 & 2.07 \\
\hline & & $\mathrm{F}$ & 1.14 & 2.28 \\
\hline \multirow{5}{*}{$\begin{array}{l}\text { Early subacute } \\
\text { period }\end{array}$} & \multicolumn{2}{|l|}{ Used equation } & Eqs.3-5 & Eqs.10,14 \\
\hline & \multirow{2}{*}{$\sum(\mathrm{E}-\mathrm{O})^{2}$} & M & 18.55 & 70.00 \\
\hline & & $\mathrm{F}$ & 27.81 & 76.43 \\
\hline & \multirow{2}{*}{$\mathrm{I}(\mathrm{E}-\mathrm{O}) \mathrm{I}$} & M & 2.85 & 5.47 \\
\hline & & $\mathrm{F}$ & 3.27 & 5.38 \\
\hline \multirow{5}{*}{$\begin{array}{l}\text { Late subacute } \\
\text { period }\end{array}$} & \multicolumn{2}{|l|}{ Used equation } & Eqs.3-5 & Eqs.11,15 \\
\hline & \multirow{2}{*}{$\sum(\mathrm{E}-\mathrm{O})^{2}$} & M & 82.79 & 2779.28 \\
\hline & & $\mathrm{F}$ & 88.32 & 2756.91 \\
\hline & \multirow{2}{*}{$\mathrm{I}(\mathrm{E}-\mathrm{O}) \mathrm{I}$} & M & 5.66 & 26.36 \\
\hline & & $\mathrm{F}$ & 6.70 & 26.71 \\
\hline \multirow{5}{*}{ Chronic period } & Used equation & & Eqs.3-5 & Eqs.12,16 \\
\hline & \multirow{2}{*}{$\sum(\mathrm{E}-\mathrm{O})^{2}$} & M & 56.34 & 82.46 \\
\hline & & $\mathrm{F}$ & 102.15 & 471.14 \\
\hline & \multirow{2}{*}{$\mathrm{I}(\mathrm{E}-\mathrm{O}) \mathrm{I}$} & M & 7.17 & 7.96 \\
\hline & & F & 9.46 & 19.66 \\
\hline
\end{tabular}

$\mathrm{M}^{*}$ : male mice; $\mathrm{F}^{* *}$ : female mice; $p$-value is greater than 0.995 for "proacent" formulas for both male and female mice, respectively. $p$-value is less than 0.05 for Gompertz formulas for both male and female mice, respectively; E: formula-derived value of mean after-survival time for male and female mice, respectively; O: Sacher's reported value of mean after-survival time for male and female mice, respectively.

to the Sacher's data, and that the "probacent" model of death rate, Eq.2 seems to be applicable and better-fitting to the reported data than the Gompertz model.

\section{DISCUSSION}

Table 1 and Figure 1 reveal a close agreement between the formula-derived values and the Sacher's-reported data on mean after-survival times (MAS) ( $p>0.995)$.

Table 2 shows that the "probacent" equations, Eqs. 3-5 have noticeably smaller least sum of squares and least maximum difference than the Gompertz equations. This finding indicates that the "probacent" model is statistically better fitting to the Sacher's data. The $p$-value is $>0.995$ for the "probacent" equations and $<0.05$ for the Gompertz equations, suggesting that the Gompertz model appears overall not applicable to the Sacher's data.

Data-points of male and female mice appear overall fall much closer to or on the solid curved line represented by the "probacent" equations than the dashed straight lines represented by the Gompertz equations in Figures 2 and 3, respectively.

The author feels that in a variety of biomedical phenomena, if Eqs.1 and $\mathbf{2}$ are applicable, the values of constants $\gamma$ and $c$ are generally greater than one or less than one but not one, indicating a curved line when plotted on a X-Y graph paper as seen Figures 1-3. The $\gamma$ and $c$ values are relatively rarely one, indicating a straight line on the graph or otherwise approximately appearing straight. This phenomenon seems to be analogous in physics to that light path is actually curved when passing through a gravitational field of space but appears straight $[44,45]$.

If the $\gamma$ value becomes equal to one, Eq.1 represents a log-normal distribution. If the c value is one, Eq.2 that is derivable from Eq.1 [33] becomes essentially similar to the Weibull distribution [1]. If the base of logarithm is one, the lognormal distribution becomes a norml distribution $\left(\log _{1} 1^{n}=n\right)[41,46]$. If the logarithm of one as its base is taken for X axis of time, the Gompertz distribution might be similar to the Weibull distribution, and so might be a specific form of the "probacent"-probability equation. It seems to the author that the "probacent" model may be applicable as a general approximation method to make useful predictions of probable outcomes in a variety of biomedical phenomenon [41,42].

Hematopoietic cells of bone marrow, intestinal tract and central nervous system are most vulnerable to radiation effects [47-52]. Body responses to lethal radiation effects reflect status of living body in which pathologic changes, physiologic repair (response and regeneration) and inherent aging process are concurrently occurring [37]. Death is caused by multi-organ failure. In case of high dose, infection and hemorrhage are earliest contributing factors to death in lethal total body irradiation, damaging most sensitive hematopoietic cells of bone marrow [37,47,52].

High dose rates (330 - $2500 \mathrm{r} /$ day) shorten mean after-survival times (MAS) in acute period, causing death within two weeks (Table 1). Decrease in dose rates increases MAS in mice. Cui and his coworkers demonstrated that a fractionated total body irradiation (FTBI) increased survival rates and therapeutic effects of FTBI in bone marrow transplantation in mice [53]. This finding is remarkably illustrated by the curved and prolonged line of MAS in the chronic period of very low dose rates (Figures 1-3). 
Biologic responses to lethal radiation effects are dependent on radiation dose rate and duration of exposure, and are reflected in survival times. The values of constants, $a, b$ and $c$ of Eq.2 of death rate seem to be determined by the underlying status of biologic responses.

The "probacent" model and findings in this study might be hopefully helpful in research to investigate human tolerance to low dose rates for long durations of exposure in total body irradiation, and further in research in a variety of biomedical phenomena.

\section{CONCLUSIONS}

The "probacent" model of death rate equation is applied to the Sacher's experimental data on dose rates versus mean after-survival times (MAS) in mice daily irradiated during the duration of life [37]. The Gompertz model is also applied to the data. Predictive formulas are constructed by both models and compared regarding their fit to data.

In this comparative study of which model would better fit to data, it is found that the "probacent" equations not only well fit the data but also more closely express the relationship between dose rate and mean after-survival time than the Gompertz equations. The Gompertz model appears approximately applicable in acute and chronic period (higher and lower doses) but seems to be overall not applicable.

The data-points connecting line is actually curved as seen in Figures 1-3, suggesting better fit of the curvedline expressing "probacent" model rather than the straight-line expressing Gompertz model.

The "probacent" model and findings in this study might-be hopefully helpful in research in human tolerance to low dose rates for long durations of exposure in total body irradiation, and further in research in a variety of biomedical phenomena.

\section{ACKNOWLEDGEMENTS}

The author is thankful to Dr. George A. Sacher, Argonne National Laboratory, USA for his personal advice, encouragement and his highly valuable and comprehensive work in radiation research, sending me a reprint of his article on which the author's current study is based.

The author thanks Dr. Lester Van Middlesworth for his outstanding research on radiation and thyroid, and for his personal valuable instructions and advice in my research related to thyroid physiology, using ${ }^{131}$ I radioisotopes at the Department of Physiology and Biophysics, the University of Tennessee.

I would like to express my thanks to Dr. C. W. Sheppard for his instructions and encouragement in my research related to radiation and computer programming.

\section{REFERENCES}

[1] Lee, E.T. and Wang. J.W. (2003) Statistical methods for survival data. John Wiley \& Sons, Hoboken, pp. 8-197. doi:10.1002/0471458546.ch2

[2] Heligman, L. and Pollard, J.H. (1980) The age pattern of mortality. Journal of Inst. Actuaries, 107, 49-80.

[3] Gompertz, B. (1994) Parametric models. In: Statistical methods in medical research, Blackwell Science, Cambridge, pp. 482-483.

[4] Kaplan, E.L. and Meier, P. (1958) Nonparametric estimation for incomplete observations. Journal of American Statistical Association, 53, 457-481. doi: $10.2307 / 2281868$

[5] Gordis, L. (2004) The Kaplan-Meier method. In: Epidemiology, Elsevier Saunders, Philadelphia, pp. 104-106.

[6] Mould, R.F. (1976) Calculation of survival rates by the life table and other methods. Clinical Radiology, 27, 33-38. doi:10.1016/S0009-9260(76)80011-6

[7] American Joint Commission. (1983) Reporting of cancer survival and results. In: Manual for Staging of Cancer. Lippincott, New York, pp. 11-21.

[8] Cox, D.R. and Oaks, D. (1985) Distribution of failure times; parametric Statistical analysis: Simple sample; single-sample nonparametric methods. In: Analysis of survival data, Chapman and Hall, London, pp. 13-61.

[9] Chung, S.J. (1960) Studies on a mathematical relationship between stress an response in biological phenomena. Republic of Korea Journal of the National Academy of Sciences, 2, 115-162.

[10] Chung, S.J. (1986) Computer-assisted predictive mathematical relationship among metrazol dose and time and mortality in mice. Computer Methods and Programs in Biomedicine, 22, 275-284. doi:10.1016/0169-2607(86)90004-0

[11] Chung, S.J. (2007) Computer-assisted predictive formulas expressing survival probability and life expectancy in US adults, men and women, 2001. Computer Methods and Programs in Biomedicine, 86, 197-209. doi:10.1016/i.cmpb.2007.02.009

[12] Chung, S.J. (2011) Predictive formulas expressing relationship among dose rate, duration of exposure and mortality probability in total body irradiation in humans. Journal of Biomedical Science and Engineering, 4, $497-$ 505. doi:10.4236/jbise.2011.47063

[13] Chung, S.J. (1959) Studies of positive radial acceleration on mice. Journal of Applied Physiology, 14, 52-54.

[14] Boak, H. and Chung, S.J. (1962) Studies on a relationship between dose, time and percentage of occurrence of response and a method of evaluation of combined action in drugs. The New Medical Journal, 5, 35-82.

[15] Kim, C.C. and Chung, S.J. (1962) Studies on a relationship between stress, duration of exposure and percentage of response in goldfish to single, double, and triple stresses of acceleration, electroshock, heat, chemical and osmotic stimuli. Republic of Korea Theses of Catholic Medical College, 5, 257-336.

[16] Cho, D.W. and Chung, S.J. (1961) Studies of tolerance of Paramecium caudatum to hydroxyl ions. Bulletin of $\mathrm{Ya}$ maguchi Medical School, 8, 151-160.

[17] Chung, S.J. (1989) Computer-assisted mathematical relationship among electroshock voltage and duration and occurrence of convulsion in mice. Computer Methods and Programs in Biomedicine, 28, 23-30. doi:10.1016/0169-2607(89)90177-6 
[18] Cerveny, T.J., MacVittie, T.J. and Young, R.W. (1989) Acute radiation syndrome in humans. In: R. J. Walker and T. J. Cerveny, Ed., Medical consequences of nuclear warfare, TMM Publishers, Office of the Surgeon General, Falls Church, Virginia, 15-36.

[19] Forbes, W.H., Sergent, F. and Roughton, F.J.W. (1988) The risk of carbon monoxide uptake by normal men. American Journal of Physiology, 143, 594-608.

[20] Chung, S.J. (1988) Formula predicting carboxyhemoglobin resulting from carbon monoxide exposure. Veterinary and Human Toxicology, 30, 528-532.

[21] Prescott, L.F. Roscoe, P., Wright, N. and Brown, S.S. (1991) Plasma paracetamol half-life and hepatic necrosis in patients with paracetamol overdosage. Lancet, 1, 519522.

[22] Chung, S.J. (1989) Computer-assisted predictive mathematical relationship among plasma acetaminophen concentration, time after ingestion and occurrence of hepatotoxicity in man. Computer Methods and Programs in Biomedicine, 28, 37-43. doi:10.1016/0169-2607(89)90179-X

[23] Chung S.J. (1991) Formula predicting survival in patients with invasive malignant melanoma. International Journal of Biomedical Computing, 28, 151-159. doi:10.1016/0020-7101(91)90051-F

[24] Chung, S.J. (1994) Formula expressing relationship among lesion thickness, time after diagnosis and survival probability in patients with malignant melanoma. International Journal of Biomedical Computing, 37, 171-180. doi:10.1016/0020-7101(94)90139-2

[25] Chung, S.J. (1993) Formula predicting survival probability in patients with heart transplantation. International Journal of Biomedical Computing, 32, 211-221. doi:10.1016/0020-7101(93)90015-X

[26] Magbool, G., Kaul, K.K., Corea, J.R., Osman, M. and Atfaj, A. (1993) weight and height in Saudi children six to 16 years from the Eastern Province. Annals of Saudi Medicine, 13, 344-349.

[27] Chung, S.J. (1994) Formulas expressing relationship among age, height and weight, and percentile in Saudi and US children of ages of 6-16 years. International Journal of Biomedical Computing, 37, 258-272. doi:10.1016/0020-7101(94)90124-4

[28] Sholz, D.C., Kitzman, D.W., Hagen, P.T., Ilstrup, D.H. and Edwards. W.D. (1998) Age-related changes in normal heart during the first 10 decades of life, Part I. (Growth): A quantitative anatomic study of 200 specimens from subjects from birth to 19 years old. Mayo Clinic Proceedings, 13, 126-136, 637.

[29] Chung, S.J. (1990) Formulas predicting the percentiles of heart weight by body weight in subjects from birth to 19 years of age. International Journal of Biomedical Computing, 26, 257-269. doi:10.1016/0020-7101(90)90049-Z

[30] Chung, S.J. (1990) Formulas predicting the percentile of serum cholesterol levels by age in adults. Archives of Pathology and Laboratory Medicine, 114, 869-895.

[31] Chung, S.J. (1992) Relationship among age, serum cholesterol level and population percentile in adults. International Journal of Biomedical Computing, 31, 99-116. doi:10.1016/0020-7101(92)90066-2

[32] Arias, E. (2004) United States life Tables, 2001. National Vital Statistics Report, 52, 1-40.
[33] Chung, S.J. (1995) Formulas expressing life expectancy, survival probability and death rate in life tables at various ages in US adults. International Journal of Biomedical Computing, 39, 209-217. doi:10.1016/0020-7101(94)01068-C

[34] Chung, S.J. (1997) Comprehensive life tables of computer-assisted predictive mathematical relationship among age and life expectancy, survival probability or death rate in US adults. Computer Methods and Programs, 52, 67-73. doi:10.1016/S0169-2607(96)01778-6

[35] Mehta, S.C. and Joshi, H.C. (2004) Model based point estimates of survival/death rate: An input for radiation risk evaluation in Indian context. Indian Journal of $\mathrm{Nu}$ clear Medicine, 19, 16-18.

[36] Sacher, G.A. (1956) On the statistical nature of mortality, with especial reference to chronic radiation mortality. Radiology, 67, 250-257.

[37] Sacher, G.A. (1959) On the radiation of lethality to radiation injury, and its relevance for the prediction problem. 9th International Congress of Radiology, München, 2330 December 1959.

[38] Travis, E.L., Peters, L.J., Thames, H.D., et al. (1985) Effect of dose-rate on total body irradiation: Lethality and pathologic findings. Radiology and Oncology, 4, 341-351. doi:10.1016/S0167-8140(85)80122-5

[39] Ellington, F. (1947) Influence of dose fractionation on the lethal X-ray effect produced by total body irradiation in mice. Radiology, 47, 238-241.

[40] Grahn, D. (1958) Acute radiation response of mice from a cross between radio-sensitive and radio-resistant strains. Genetics, 43, 835-843.

[41] Chung, S.J. (2009) Seeking a New World: A New Philosophy of Confucius and Kim Hang. iUniverse, Bloomington, 68-76,153.

[42] Chung, S.J. (2011) Computer program of nonlinear, curved regression for "probacent"-probability equation in biomedicine. Journal of Biomedical Science and Engineering, 4, 620-630. doi:10.4236/jbise.2011.49078

[43] Dixon, W.J. and Massey Jr., F.J. (1957) Introduction to Statistical; Analysis, McGraw-Hill, New York, 191-227.

[44] Einstein, A. (1905) The special theory of relativity. In: S. W. Hawking, A Brief History of Time, Bantam Books, New York, 1-61.

[45] Suplee, C (1999) Physics in the $20^{\text {th }}$ Century, Hany N. Abrahams, Inc., New York, 82-180.

[46] Chung, S.J. (2010) The book of right change: A New Philosophy of Asia. iUniverse, Bloomington, 10.

[47] Warren, S. (1961) The pathology of ionizing radiation, Charles C. Thomas Publisher, Springfield.

[48] Li, X.H., Fu, D., Latif, N.H., et al. (2010) $\delta$-tocotrienol protects mouse and human hematopoietic progenitors from $\gamma$-irradiation through extracellular signal-regulated kinase/mammalian target of rapamycin signaling. Haematologica, 95, 1996-2004. doi:10.3324/haematol.2010.026492

[49] Komarova, E.A., Kondratov, R.V., Wang, K., et al. (2004) Dual effect of $\mathrm{p} 53$ on radiation sensitivity in vivo: $\mathrm{p} 53$ promotes hematopoietic injury, but protects from gastro-intestinal syndrome in mice. Oncogene, 23, 32653271. doi:10.1038/sj.onc. 1207494

[50] Department of Radiology, University of Illinois (1998) Whole body irradiation: Lesson from Chernobyl. 
www.uic.edu/com/uhrd/manuscript/section $4 /$ section $4 . h t$ $\underline{\mathrm{ml}}$.

[51] Jones, T.D., Morris. M.D., Wells, S.M. and Young, R.W. (1986) Animal mortality resulting from uniform exposures to photon radiations: Calculated $\mathrm{ld}_{50}$ and a compilation of experimental data, Oak Ridge National Laboratory, Oak Ridge.

[52] Donnelly, E.H., Nembauser, J.B., Smith, J.M., et al.
(2010) Acute radiation syndrome: Assessment and management. Southern Medical Journal, 103, 541-544. doi:10.1097/SMJ.0b013e3181ddd571

[53] Cui, Y. Yang, G., Fan, T., et al. (2002) Optimal protocol for total body irradiation for allogeneic bone marrow transplantation in mice. Bone Marrow Transplantation, 30, 843-849. doi:10.1038/sj.bmt.1703766

\section{APPENDIX}

A formula expressed by Eq.2 representing the mean after-survival time (MAS) as a function of daily dose rate in total body irradiation in mice is assumed to be applicable here to the Sacher's data [37] on the basis of the aforementioned findings.

General Formulas of the Mean After-Survival Times in Acute Period for Male Mice (Up to Two Weeks after Irradiation of Dose Rate, 2500 - 330 r/day)

Two sets of data on dose rate $(R)$ and mean after-survival time $(T)$ from the Sacher's reported values are used to determine values of $a, b, c$ in Eq.2.

1) $R=1650 \mathrm{r} /$ day of dose rate, and $T=5.4$ days of mean after-survival time.

2) $R=330 \mathrm{r} /$ day of dose rate, and $T=13.37$ days of mean after-survival time.

$$
\begin{gathered}
(\log 1650)^{c}=a+b \log 5.4 \\
(\log 330)^{c}=a+b \log 13.37
\end{gathered}
$$

The values of constants, $a$ and $b$ are derived from Eqs. A.1 and A.2 as expressed by Eqs.A.3 and A.4, respec- tively.

$$
\begin{aligned}
& a=2.86009 \times 3.21748^{c}-1.86009 \times 2.51851^{c} \\
& b= 2.53975 \times\left(2.51851^{c}-3.21748^{c}\right) \\
&(\log R)^{c}= 2.86009 \times 3.21748^{c}-1.86009 \times 2.51851^{c} \\
&+2.53975 \times\left(2.51851^{c}-3.21748^{c}\right)
\end{aligned}
$$

In order to determine the best fitting value of constant $c$, the author employs the method of the least sum of squares that is described in the author's previous publication [42]. A very close and best agreement is found between the computer-derived and the Sacher's-reported mean after-survival times with the c-value of 0.1 determined by the above method. The $a, b$ an $c$ values in the Eqs.3-5 for acute period in male mice are finally determined.

Values of constants, $a, b$ and c in Eqs.3-5 for the other three periods, early subacute, later subacute and chronic period are likewise determined.

Formulas of the Gompertz equations, Eqs.6-16 are likewise constructed from two sets of data in each of four periods. 